\title{
Disability caused by post-stroke visual impairment as an underestimated clinical problem - case report
}

\author{
Dariusz Baranowski', Konrad Rejdak', Ewelina Cisek ${ }^{2}$, Agnieszka Cisek², Agnieszka Kiszka², \\ Katarzyna Nowomiejska ${ }^{2}$, Robert Rejdak ${ }^{2}$ \\ 'Department of Neurology, Medical University of Lublin, Poland \\ ${ }^{2}$ Department of General Ophthalmology, Medical University of Lublin, Poland
}

\begin{abstract}
Ischaemic cerebral stroke is a common complication following cardiovascular diseases. In everyday clinical practice, visual field defects are diagnosed in approximately one in four ischaemic stroke patients. The incidence of cerebral stroke manifesting only with visual defects is higher than might be expected. Post-stroke visual impairment is associated with deteriorated quality of life of the affected, can lead to partially reduced self-reliance, and imposes job changes. We present a case of a 46-year-old male with visual field impairment secondary to cerebral ischaemia, to highlight this important issue.
\end{abstract}

KEY WORDS: vision problems, ischaemic stroke, disability level

Ophthalmol J 2016; Vol. 1, No. 3, 103-108

\section{INTRODUCTION}

Ischaemic cerebral stroke is a common complication of cardiovascular diseases. It is defined as a sudden onset of neurological deficits (global or focal) resulting from impaired blood flow to the central nervous system (CNS). Moreover, it is the most common cause of disability in adults.

In everyday clinical practice, visual field defects are diagnosed in approximately one in four ischaemic stroke patients [1]. The incidence of cerebral stroke manifesting only with visual field defects is higher than might be expected [2]. Vision is one of the most important human senses, and its impairment causes disability comparable to motor dysfunction of the limbs with secondary gait or posture disorders [3]. Post-stroke visual impairment is associated with markedly deteriorated quality of life leading to partially reduced self-reliance. Insecure walk caused by vision impairment can also lead to increased risk of secondary falls resulting in traumas with all of their serious complications, i.e. immobilisation after bone fractures can cause potential life-threatening pulmonary embolism episodes [4].

Post-stroke visual field defects should attract more attention of clinicians, which could significantly improve the quality of care of the affected patients. Our case of a patient with limited visual field secondary to cerebral ischaemia is aimed at highlighting this important issue.

\section{CASE REPORT}

A 46-year-old male patient was admitted to the Department of Neurology due to a suspicion of developing cerebral stroke. The first symptoms of CNS ischaemia were severe headache and bilateral deterioration of vision. The symptoms started when the patient had been playing squash. Since no other 


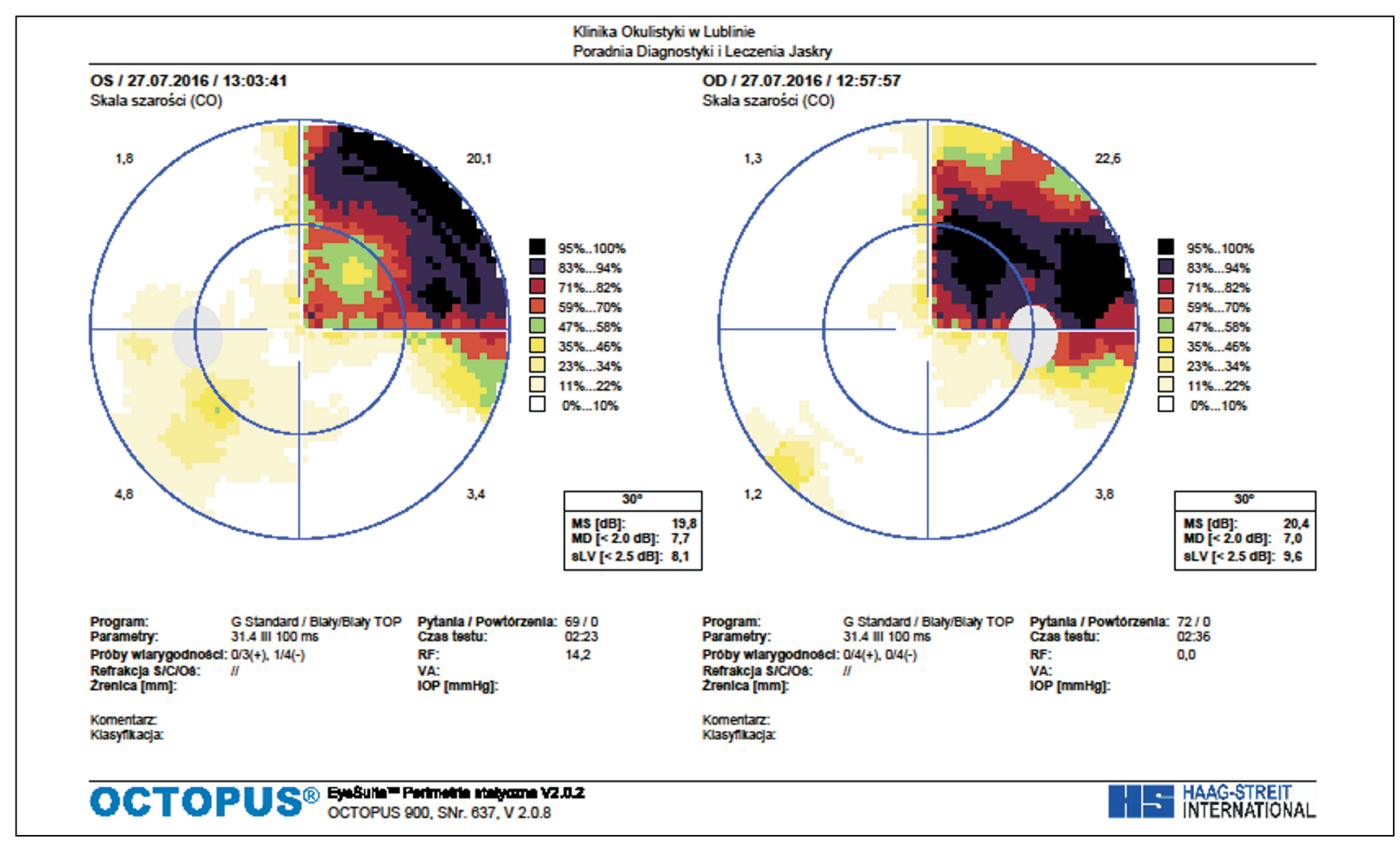

FIGURE 1. Homonymous quadrantanopia in static perimetry

symptoms occurred the patient initially headed to the Department of Ophthalmology, where the ophthalmologist excluded the pathology of the eyeball as a cause of visual field defects reported by the patient and suspected neurological disorders. Visual acuity was full in both eyes, anterior and posterior segments were normal, and pupil reactions were also normal. Static perimetry (Octopus 900, Haag-Streit, Switzerland) revealed right - upper homonymous quadrantanopia (Fig. 1). Ocular coherence tomography (Cirrus, Zeiss, US) of the macula and peripapillary retinal nerve fibre layer was within normal limits (Fig. 2). The patient was sent to the Neurological Department.

The neurological evaluation on admission revealed isolated right homonymous upper quadrantanopia (the NIHSS score only 1 point). Although there was no limb paresis, the patient walked insecurely, occasionally had to stop and turn his head trying to see and remember obstacles. Prior to cerebral stroke, the patient did not show distinct disability (the modified Rankin scale score - 0 point). Cerebral stroke resulted in disability to carry out all previous activities due to visual impairment (mRS -2 point), especially considering that the patient's profession is dentistry.

Routine computed tomography (CT) head scan did not reveal any pathology that could be respon- sible for the clinical symptoms. Neuroimaging was extended using MRI, which allowed visualisation of an ischaemic lesion located in the left occipital lobe in the brain area supplied by the left posterior cerebral artery, which corresponded to the clinical view (Fig. 3). The region showed features of water diffusion restriction in diffusion-weighted imaging (DWI) sequences, which confirmed that the lesion was subacute and new.

At the moment of admission to the stroke unit the therapeutic window for safe thrombolytic therapy was close to an end. Furthermore, an ischaemic lesion had been already formed, which had been confirmed in the MRI examination. Potential successful reperfusion after intravenous administration of r-tPA in that time would cause high risk of secondary haemorrhagic transformation of ischaemic lesion without viable chance for symptoms withdrawal.

To determine the aetiology (potential risk factors) of cerebral stroke, the patient underwent several additional examinations. Duplex Doppler ultrasound assessing the cerebral arteries in the intracranial segments visualised an occlusion of the left posterior cerebral artery, substantially impairing the blood flow to the brain (second grade in TIBI classification). A control examination taken five days later showed normal haemodynamic parameters of blood flow in the previously occluded artery, 


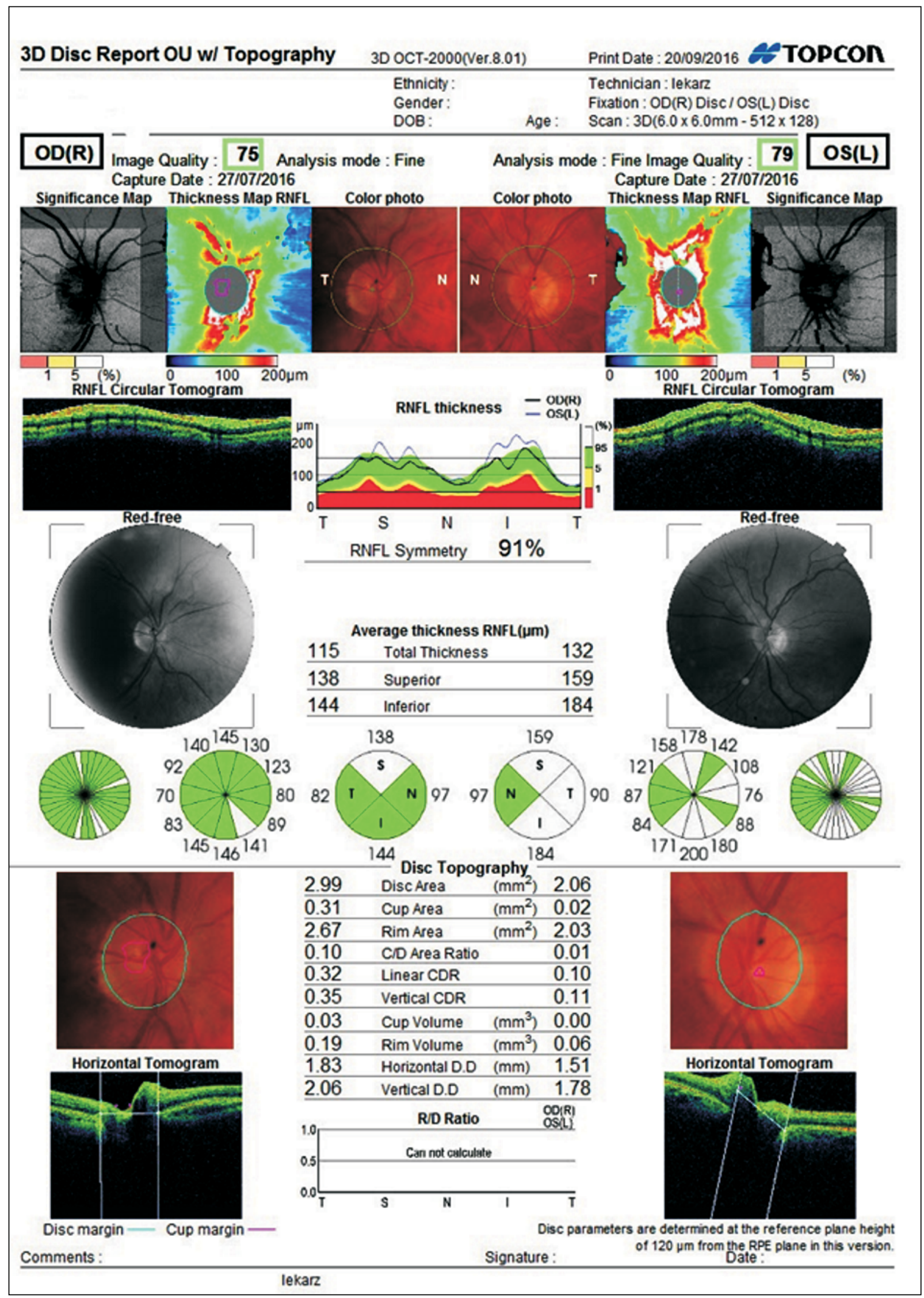

FIGURE 2. Optical coherence tomography of the peripapillary nerve fibre layer of both eyes 

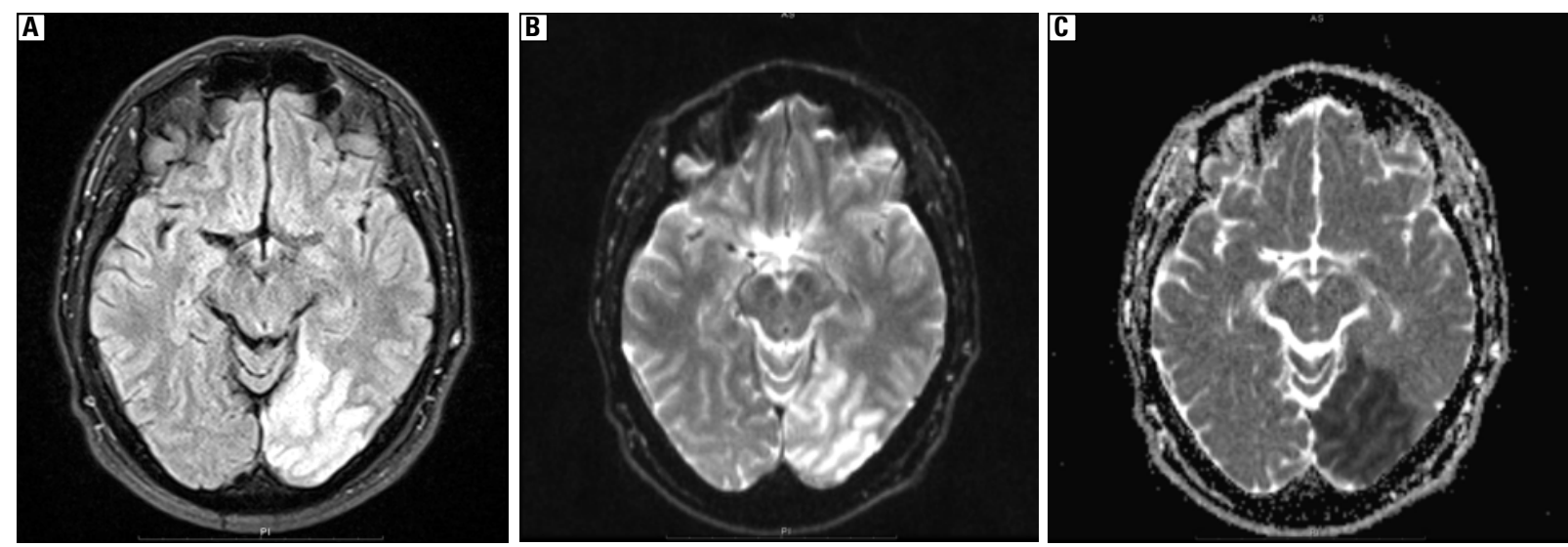

FIGURE 3. The region of ischaemia involving the left occipital lobe in T2 tirm (A), DWI (B) and ADC (C) sequences

which can mean that the occlusion was made by a clot that spontaneously dissolved. Considering the fact that symptoms occurred during squash playing (fast neck turns) the possible cause of stroke could be a cerebral artery dissection, but the MR Fat-Sat imaging sequence did not suggest any presence of artery wall haematoma.

In searching for a cardioembolic origin of the embolus, additional cardiological diagnostic examinations were carried out. Echocardiography did not reveal any valvular defects or myocardial contractility disorders; moreover, 24-hour Holter ECG monitoring did not disclose significant arrhythmias or conduction disorders. Arterial blood pressure during Holter monitoring wasn't significantly elevated. Moreover, the circadian rhythm was physiological with normal decrease observed at night (non-dipper rhythm could be an independent risk factor of vascular incidents). The laboratory tests showed mild disorders of lipid metabolism without any other abnormalities.

Considering the patient's young age additional laboratory tests towards rare blood increased coagulability states were carried out but without findings (d-dimer level, homocysteine level, antiphospholipid antibodies, anticardiolipin antibodies, antinuclear antibodies, antineutrophil cytoplasmic antibodies, factor V Leiden mutation, protein $\mathrm{C}$ and $\mathrm{S}$ level, antithrombin III level).

In conclusion, we diagnosed embolic stroke of undetermined source (esus) and planned control examinations in the future.

\section{DISCUSSION}

Ischaemic cerebral stroke is a common complication of cardiovascular diseases. It is defined as a sudden development of neurological deficits due to impaired blood flow to the central nervous system. The major aetiological factors of ischaemic cerebral stroke based on the Trial of Org 10172 in Acute Stroke Treatment (TOAST) are presented in Table $1[5,6]$.

At present, the acute stage of ischaemic cerebral stroke is managed with intravenous thrombolytic treatment to restore the blood supply to the ischaemic brain region. Thrombolysis involves the administration of a recombinant tissue plasminogen activator (rt-PA), which activates the endogenous process of fibrinolysis (degradation of fibrin that the thrombus is built of) and leads to thrombus / embolus defragmentation. The treatment is applied up to 4.5 hours after the onset of neurological symptoms of stroke (ECASS-3 study conclusions) [2, 7]; when contraindicated or in cases of ineffective rtPA, mechanical thrombectomy can be performed until six hours have elapsed, which is available in some stroke centres. During thrombectomy, a neurologist or an interventional radiologist removes the thrombus using a special intravascular device. After the end of a therapeutic time window according to recommendations we can use antiplatelet drugs, hypotensive drugs and statins.

When intensive treatment of acute ischaemic cerebral stroke is not effective or it cannot be taken (because of the end of the therapeutic window or contraindications), then residual neurological deficit symptoms following cerebral stroke are established. Necrosis of nervous cells during ischaemic stroke leads to disorders of consciousness, speech or orientation, limb paresis, and impaired sensations. When the ischaemic region involves the areas responsible for conduction or integration of visual stimuli sent to the brain from the eye, stroke leads 
Table 1. Risk factors of ischaemic cerebral stroke according to the Trial of Org 10172 in Acute Stroke Treatment (TOAST) with authors' comments

Disease of large cerebral vessels - atheromatous plaque causing substantial stenosis of blood vessels supplying the brain

Disease of small cerebral vessels - damage to the fine perforating branches supplying the cerebral subcortical structures predominantly associated with uncontrolled arterial hypertension and uncontrolled diabetes mellitus

Cardioembolic aetiology that is second to the formation of clots of blood accumulated in the heart cavities during:

- atrial fibrillation

- valve stenosis (mitral, aortic)

- post-infarction disorders of myocardial contractility

- congestive heart failure linked to different types of cardiomyopathies

Clots undergo defragmentation, which causes peripheral emboli flowing with blood stream to the brain and causing ischaemic stroke episodes

Stroke of another known but rare aetiology:

- cerebral artery dissection

- cerebral vasculitis in the course of rheumatoid diseases

- vasospasm as a complication after subarachnoid haemorrhage episode

- increased coagulation predisposing to cerebral stroke resulting from:

- antiphospholipid syndrome

- congenital mutations of gene-encoding proteins responsible for blood clotting

— neoplastic diseases

- oral contraceptive/hormone replacement therapy

- brain ischaemia caused by prolonged migraine attack

- extremely rare genetic diseases, e.g.:

- CADASIL - cerebral autosomal dominant arteriopathy with subcortical infarcts and leukoencephalopathy

- MELAS - mitochondrial myopathy, encephalopathy, lactic acidosis, stroke-like episodes;

Cryptogenic strokes whose aetiology is difficult to establish

to visual field deficits. The most common visual disorders resulting from vascular-cerebral incidents found during routine clinical practice include $[1,8]$ :

- ptosis resulting from denervation of the upper lid elevator due to damage to the oculomotor nerve supplying it;

- impaired motility of eyeballs resulting in diplopia caused by damage to the cranial nerve nuclei supplying orbital muscles, medial longitudinal fasciculus connecting their nuclei in the brainstem or corticonuclear tracts;

- paresis or paralysis of the conjugate gate of the eyeball opposite to the damaged centre coordinating conjugate eyeball movements, which is situated in the frontal lobe of the brain. The affected patient "looks towards the damaged brain hemisphere". This symptom is usually observed in the early stage of cerebral stroke; its persistence is considered a poor prognostic factor;

- bitemporal haemianopia predominantly in injures involving the optic chiasm;

- homonymous haemianopia contralateral to the location of an ischaemic lesion involving optic radiation;
- superior and inferior quadrantanopia contralateral to the partial damaged involving optic radiation;

- homonymous haemianopia contralateral to the lesion with central part sparing in visual cortex injuries;

- visual extinction (no visual field defects or impaired visual acuity yet the patient unconsciously ignores the stimuli occurring in certain areas of the visual field and focuses on the objects in its remaining parts) resulting from dysfunction of many cortical and subcortical structures.

In the majority of clinical studies and in everyday clinical practice, the extent (the degree of deficit) of neurological symptoms developing during cerebral stroke is estimated according to the National Institutes of Health Stroke Scale (NIHSS). The scale is used to qualify patients for acute stroke treatment and to evaluate its efficacy. Unfortunately, NIHSS does not always reflect the degree of post-stroke disability. A patient with isolated visual field impairment (as the only consequence of cerebral stroke) is scored 1 or 2 , although he or she cannot efficiently read, operate a computer, or safely drive a car. Therefore, the extent 
of disability caused by neurological deficits is assessed using the modified Rankin's Scale (mRS) providing more accurate data, which significantly affects further neurological and ophthalmic management.

In stroke patients, diagnostic procedures and optimal control of stroke risk factors are essential and enable quick secondary prophylactic interventions to prevent another vascular incident and progression of disability [2, 6]. Moreover, simultaneous rehabilitation should be carried out to limit the disability resulting from a residual neurological deficit [9]. Similar to the cases of logotherapy in patients with speech disorders or early kinesitherapy in patients with motor dysfunction, visual impairments require complex management [9-12]. It is essential that patients with visual disorders are thoroughly examined by a neurologist and ophthalmologist and undergo follow-up neuroimaging visualising and assessing the extent of cerebral stroke (preferably using MRI) as well as perimetry tests [13-15]. Complex medical attention, objectivisation of disorders, and evaluation of prognosis favourably affect the patient's mental condition, which prevents reactive mood disorders enhancing disability [16]. The university centres we are employed in cooperate to provide patients who have post-stroke visual impairments as the prevailing neurological symptom following stroke with complex care. We believe that the presented model of cooperation is worth recommending and implementing in other stroke centres.

\section{FINANCIAL DISCLOSURE}

None declared.

\section{CONFLICT OF INTEREST}

None declared.

\section{REFERENCES}

1. Sand KM, Wilhelmsen G, Næss H, Midelfart A, Thomassen L, Hoff JM. Vision problems in ischaemic stroke patients: effects on life quality and disability. Eur J Neurol 2015; 23: 1-7.

2. Lawlor M, Richard Perry R, Hunt BJ, Plant GT. Strokes and vision: The management of ischemic arterial disease affecting the retina and occipital lobe. Surv Ophthalmol 2015; 60: 296-309.

3. Langelaan M, de Boer MR, van Nispen RM et al. Impact of visual impairment on quality of life: a comparison with quality of life in the general population and with other chronic conditions. Ophthalmic Epidemiol 2007; 14: 119-126.

4. Lord SR, Dayhew J. Visual risk factors for falls in older people. J Am Geriatr Soc 2001; 49: 508-515.

5. Chen P-H, Gao S, Wang Y-J, Xu A-D, Li Y-S, Wang D. Classifying Ischemic Stroke, from TOAST to CISS. CNS Neurosci Ther 2012; 18: 452-456.

6. Arboix A. Cardiovascular risk factors for acute stroke: Risk profiles in the different subtypes of ischemic stroke. World J Clin Cases 2015; 3: 418-429.

7. Gurewich V. Thrombolysis: A critical first-line therapy with an unfulfilled potential. Am J Med 2016; 129: 573-575.

8. Sand KM, Midelfart A, Thomassen L. Visual impairment in stroke patients - a review. Acta Neurol Scand 2013; 196 (Suppl.): 52-56.

9. Jones SA, Shinton RA. Improving outcome in stroke patients with visual problems. Age Ageing, 2006; 35: 560-565.

10. Rowe $F$, Brand $D$, Jackson CA. Visual impairment following stroke: do stroke patients require vision assessment? Age Ageing 2009; 38: 188-193.

11. Pollock $A$, Hazelton $C$, Henderson CA. Interventions for visual field defects in patients with stroke. Stroke 2012; 43: e37-e38.

12. Pollock A, Hazelton C, Henderson CA. Interventions for disorders of eye movement in patients with stroke. Cochrane Database Syst Rev 2011; 10: CD008389.

13. Kurz KD, Ringstad G, Odland A, Advani R, Farbu E, Kurz MW. Radiological imaging in acute ischaemic stroke. Eur J Neurol 2015; 23: 8-17.

14. Kim BJ, Kang HG, Kim H-J et al. Magnetic Resonance Imaging in Acute Ischemic Stroke Treatment. J Stroke 2014; 16: 131-145.

15. Brown HDH, Woodall RL, Kitching RE, Baseler HA, Morland AB. Using magnetic resonance imaging to assess visual deficits: a review. Ophthal Physiol Opt 2016; 36: 240-265.

16. Jones GC, Rovner BW, Crews JE, Danielson ML. Effects of depressive symptoms on health behavior practices among older adults with vision loss. Rehabil Psychol 2009; 54: 164-172. 